\section{The power law for the perception of rotation by airline pilots*}

\author{
BRANT CLARK \\ San Jose State College, San Jose, California 95114 \\ and \\ JOHN D. STEWART \\ Ames Research Center, NASA, Moffett Field, California 94035
}

The purpose of this study was to determine the power laws for the perception of rotation about the three major body axes. Eighteen airline pilots made magnitude estimates of 5-sec pulses of nine angular accelerations having a range of acceleration $x$ time of $10-150 \mathrm{deg} / \mathrm{sec}$. The results showed that (1) the power law with an exponent of 1.4 describes the subjective motion of these pilots for all three major body axes, (2) the power law also describes the perception of motion for individual pilots with a substantial range of exponents, (3) there were significant correlations among the exponents for the three body axes, and (4) the data suggest that the power law over the wide range used may be more complex than implied by a formula with a single exponent.

Studies of the perception of rotation during angular acceleration have been, for the most part, limited to rotation of the head and body about the cephalocaudal ( $z$ or yaw) axis. Consequently, data on the perception of rotation applied about the O's somersaulting (pitch or $y$ ) axis and cartwheeling (roll or $x$ ) axis are extremely limited (Clark \& Stewart, 1970). Similarly, studies of magnitude estimates of suprathreshold angular accelerations are even more limited. Elsner (1971) has demonstrated that, with $O$ seated erect and rotating about an earth-vertical axis, Stevens's power law describes the increase in perceived motion over an acceleration $x$ time (at) range from 10 to $150 \mathrm{deg} / \mathrm{sec}$. Elsner also reported that the exponent for the perception of rotation is slightly greater than that for the oculogyral illusion. Reason and Graybiel (1969) have shown that magnitude estimates of generalized Coriolis sensations (produced by tilting the head $30 \mathrm{deg}$ from the vertical while rotating between 6 and $16 \mathrm{rpm}$ ) increased as a power function of the angular velocity. Their exponents, however, were found to be substantially greater than those reported by Elaner for simple rotations about the earth-vertical axis while the $O$ was seated erect. These two studies produced quite different stimulation of the semicircular canals, and a comparison of the results suggests that the vestibular system may operate in different ways for different types of suprathreshold stimuli. This may be

\footnotetext{
*The experimental work for this study was carried out at Ames Research Center under National Aeronautics and Space Administration Grant NGL 05-046-002 to San Jose State College.
}

true in spite of the fact that the average sensitivities to angular accelerations about the three body axes are very similar (Clark \& Stewart, 1970). The larger exponents reported by Reason and Graybiel may also be a result of the smaller range of stimuli used (Poulton, 1968). A final variable that probably contributes to the differences between these two studies is that Reason and Graybiel were interested in the general effects of Coriolis accelerations, and consequently they asked their $\mathrm{OB}_{3}$ to report on the general effects of head motion rather than on body or target motion per se. investigations at Ames Research Center designed to determine how pilots process rotary information in simulators and in flight. It was the specific purpose of the present study to determine the power laws for the perception of rotation about the three major body axes for airline pilots.

\section{METHOD}

Apparatus

The Ames Man-Carrying Rotation Device (MCRD) was used to rotate the O. (Clark \& Stewart, 1970). This device rotates about an earth-vertical axis with minimum vibration, and angular accelerations can be programmed within narrow limits of error. The rise time of the accelerations is of the order of 0.1 sec. Since the MCRD rotated only about an earth-vertical axis, two special seats were used to place the $O$ in the proper position at the center of rotation to rotate his head about its $x, y$, or $z$ axis. In each case, the $O$ 's head was positioned at the center of rotation and his leg were drawn up in a sitting position. To rotate him about his $z$ axis, the $O$ was placed in a normal
This study is part of a series of erect, seated position. To rotate him about his $x$ axis, a chair was laid horizontally so the $O$ was flat on his back with his legs in a seated position. This produced the same angular acceleration that would be found in a roll. To rotate the $O$ around his $y$ axis, he was rotated $90 \mathrm{deg}$ from the previous position so that his right ear was down. Thus, with the simulator rotating about an earth-vertical axis, the $O$ was turned around his pitching axis. These unusual positions were necessary to maintain the direction of the $g$ vector constant during rotation about the $x$ and $y$ axes.

\section{Observers}

The Os were 18 airline pilots who were in good health by their own affirmation and by a current Class I F A A Medical Certificate. Their hearing was normal, and their thresholds about the $z$ axis as measured in an earlier study fell within the normal range (Clark \& Stewart, 1970).

\section{Procedure}

The $O$ sat or reclined in the seat with his helmet preseed firmly into a U-shaped headrest for rotations about the $x$ and $z$ axes, while for rotation about the $y$ axis, the helmet was secured in position. All observations were made in complete darkness. The accelerations were presented for $5 \mathrm{sec}$ following a ready signal, and the nine stimulus (at) levels were those used by Elsner (1971). These were: at $=10$, $15,25,40,60,75,90,120$, and $150 \mathrm{deg} / \mathrm{sec}$; the corresponding intertrial intervals, derived from Parsons's (1970) study, were: $1,1,2$, $2,2,2.5,3,3$, and $3 \mathrm{~min}$. The accelerations were presented in random order throughout each condition, each level being presented six times, three in each direction in random order. The standard stimulus (at $=60 \mathrm{deg} / \mathrm{sec}$ ) was presented twice, once in each direction before each session began. The direction of the acceleration was varied systematically within blocks of nine trials to maintain the velocity of the MCRD within $\pm 30 \mathrm{rpm}$. Each 25-min session involved 11 trials, including the two standard stimuli. An initial practice block of trials was used to familiarize the $O$ with the procedure, but these data were not used in the computations. All of the data were collected for each $O$ at a given body position before data were collected for the other conditions. The order of the collection of data for body position was systematically ordered among $O$ s using a Latin-square procedure to reduce order effects.

The O's task following each acceleration was to give a magnitude estimate of his perceived rate of spin 


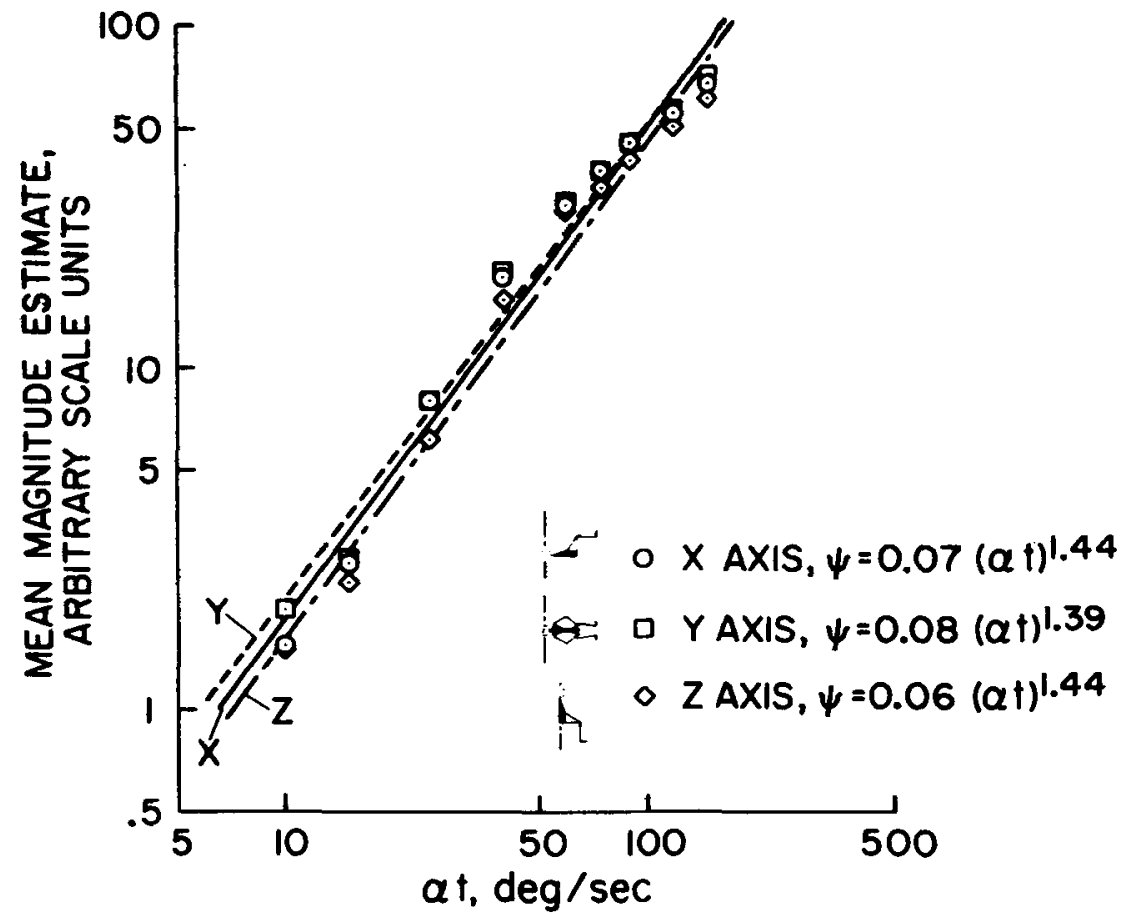

Fig. 1. Geometric mean magnitude estimates of 18 pilots for the $x, y$, and $z$ body axes.

using a ratio scaling procedure (Elsner, 1971; Stevens, 1956). They also reported the end of the aftereffect, but these data are not related to the power law and will be reported elsewhere. The $O$ s were given detailed instructions regarding the use of the procedure of magnitude estimation and practice in using ratio scales. They were instructed to choose a modulus (or base number) between 10 and 100 following the first standard acceleration (at $=60 \mathrm{deg} / \mathrm{sec}$ ) that would be the most comfortable and meaningful and make the ratio judgments easy. The second standard stimulus was then presented in the opposite direction, and the $O$ was informed that it was at the same level. Following these standard trials, the Os were presented with the nine test accelerations in a practice series and they made magnitude estimates using their ratio scale. They were not permitted to respond with zero, but decimals were permitted. The $O$ responded with his magnitude estimate on command, $5 \mathrm{sec}$ after the termination of the acceleration. It was emphasized that his magnitude estimates were to be based on his own perception of his rate of spin rather than on extraneous cues or the amount of displacement of the cab.

\section{RESULTS}

Results Averaged Across 18 Pilots

Each pilot in this study chose the modulus that was most comfortable and meaningful for him. Consequently, the data for each $O$ were averaged, using the method devised by Lane, Catania, and Stevens (1961), and adjusted grand geometric mean magnitude estimates were determined for the 18 pilots for each stimulus level (Fig. 1). The plots of these data show an increase in subjective motion represented by a near straight line function with a reduction in slope at the upper levels, but no threshold effect. The resulting exponents for the three axes were found to be very similar (Table 1 ). In fact, the values of the exponents turned out to be identical to the second decimal place for the $x$ and $z$ axes. These results show that the pilots' subjective response to angular accelerations follows a power law with an exponent of approximately 1.4. Therefore, the perception of rotation exhibits an "expander action" (exponent greater than 1) that is greater than that of most sensory systems (Stevens, 1970).
Power Laws for

Individual Pilots

The results for individual pilots confirm Elsner's (1971) findings that the perception of rotation for individual $O s$ is also described by a power law. The ranges of these individual exponents for the three axes were: $x=0.89$ to $2.05 ; y=0.88$ to 1.97; and $z=0.85$ to 2.30 . The correlation coefficients between the axes were: $\mathrm{xy}=+0.66 ; \mathrm{xz}=+0.74$; and $y z=+0.48$. All of these correlations were significantly greater than zero, suggesting that the individual pilots respond to rotation in much the same manner in the three different body axes.

The values of the individual exponents were also used to analyze the differences between the exponents of the three axes and between the direction of rotation in each axis. This analysis showed that the differences for the three axes were not significant ( $p>.20$ in each case). Comparisons of the individual exponents in the two directions for each axis revealed a high correlation in each axis: $x=+0.92 ; y=$ $+0.86 ; \mathrm{z}=+0.89$. Furthermore, the differences between the means in the two directions revealed small and not significant differences $(p>.05)$ for the $y$ and $z$ axes (Table 1 ). However, the mean exponent for a roll to the right was significantly greater than for $a$ roll to the left $(p<.001)$, although the absolute difference was not great.

These data may be summarized by the following statements: (1) The power functions for the perception of rotation are larger than for most other sensory systems, although the individual differences are great. (2) The power functions for the three major body axes are not significantly different, and they are very similar for both directions of rotation in the three major axes.

\section{DISCUSSION}

The results of this experiment show convincingly that the perception of rotation by aircraft pilots can be described by Stevens's power law for all three axes. For the full range of accelerations used, the exponent is a p proximately 1.4. These results confirm Elsner's (1971) finding for the $z$ axis and extend it to the other two axes. It is worth noting that the

Table 1

Exponents for the Power Laws for the Perception of Rotation for 18 Airline Pilots

\begin{tabular}{lllr}
\hline Axis & \multicolumn{2}{c}{ Direction of Motion } & Total \\
\hline & Roll Left & Roll Right & \\
$X$ (Roll) & 1.38 & 1.50 & 1.44 \\
$Y$ (Pitch) & Pitch Up & Pitch Down & \\
Z (Yaw) & 1.34 & 1.41 & 1.39 \\
\hline
\end{tabular}


correlations among the pilots' individual exponents for the three axes are moderately high in contrast with threshold values, which exhibit low, insignificant correlations among the three major body axes (Clark \& Stewart, 1970). Thus, although the threshold for rotation about one axis does not predict that about another axis, the exponent for the power law in any one axis will give a fairly good prediction of the exponents in the other axes.

The data from these 18 pilots appear to indicate that they process information resulting from stimulation by angular acceleration about one body axis in a very different manner from that used by men exposed to complex Coriolis accelerations. These accelerations produce complex, changing stimulation in more than one major axis at a time (Reason \& Graybiel, 1969). In fact, the resultant effect is a rotation of the axis and a change in magnitude of acceleration during the head movement. Reason and Graybiel reported exponents of 2.07 and 2.33 for right and left head tilts, respectively, for nine men in an "eyes closed" condition compared with an exponent of $\mathbf{1 . 4}$ for the pilots reported above. However, computations of the at values for the Coriolis accelerations used by Reason and Graybiel showed a restricted range compared with the range observed by the pilots. The lowest corresponding value for the Coriolis effect was approximately $19 \mathrm{deg} / \mathrm{sec}$ at $6 \mathrm{rpm}$, while the highest was $50 \mathrm{deg} / \mathrm{sec}$ at $16 \mathrm{rpm}$. Thus, the range of Coriolis accelerations was less than one-quarter of the range used with the pilots. These differences in range produce results that are comparable to the findings in several sensory systems reported by Poulton (1968), and make the Coriolis data quite comparable to the exponents found with the pilots. This finding is somewhat surprising since the Os' task and the subjective effects were quite different in the two experiments. The pilots were merely asked to report on the magnitude of rotation, and their subjective reports indicated that this was the effect estimated in roll, pitch, and yaw. Mild, transient nausea was reported by two or three pilots during the first run in a new position, but no marked motion sickness was reported. On the other hand, Reason and Graybiel asked their Os to report on the magnitude of the total Coriolis effect following a head movement of $30 \mathrm{deg}$ while they were rotated at a constant velocity. The Os reported no difficulty in quantifying their sensations, but they had considerable difficulty in describing the precise nature of the directional components. The most common

Table 2

Exponents, Intercepte, and Correlations for the Mapitude Estimates for the Total Data and the $U$ pper and Lower Levels

\begin{tabular}{|c|c|c|c|c|}
\hline \multirow[b]{2}{*}{ Segment } & & \multicolumn{3}{|c|}{ Axis } \\
\hline & & $\mathbf{x}$ & $\mathbf{Y}$ & $\mathbf{z}$ \\
\hline All Nine Points & $\begin{array}{l}\text { Exponent } \\
\text { Intercept } \\
\text { Correlation }\end{array}$ & $\begin{array}{l}1.44 \\
0.07 \\
0.988\end{array}$ & $\begin{array}{l}1.39 \\
0.08 \\
0.989\end{array}$ & $\begin{array}{l}1.44 \\
0.06 \\
0.990\end{array}$ \\
\hline $\begin{array}{l}\text { Lower Four Points } \\
\text { at }<60\end{array}$ & $\begin{array}{l}\text { Exponent } \\
\text { Intercept } \\
\text { Correlation }\end{array}$ & $\begin{array}{l}1.83 \\
0.02 \\
0.889\end{array}$ & $\begin{array}{l}1.89 \\
0.02 \\
0.889\end{array}$ & $\begin{array}{l}1.72 \\
0.03 \\
0.994\end{array}$ \\
\hline $\begin{array}{l}\text { Upper Five Points } \\
\text { at }>60\end{array}$ & $\begin{array}{l}\text { Exponent } \\
\text { Intercept } \\
\text { Correlation }\end{array}$ & $\begin{array}{l}0.88 \\
0.82 \\
0.897\end{array}$ & $\begin{array}{l}0.90 \\
0.78 \\
1.000\end{array}$ & $\begin{array}{l}0.86 \\
0.84 \\
0.999\end{array}$ \\
\hline
\end{tabular}

report was a sensation of banking and turning with some pitching. This difficulty has been reported by others, e.g., Stewart and Clark (1965). An additional factor that complicated the reports following Coriolis stimulation was that the Os also included "feelings of dizziness" or a "buzzing in the head" in the magnitude estimates. Indeed, one $O$ had to be dropped because of extreme motion sickness. Therefore, in spite of gross differences in the subjective effects observed in these two experiments, when the range of accelerations is taken into account, the power laws appear to be comparable.

Directional asymmetries have been reported following head movements in opposite directions during rotation by Reason and Graybiel (1969) as noted above and by Guedry and Montague (1961). There were no clear-cut directional asymmetries for simple rotation about the three major body axes, with the possible exception of the pitch axis. However, these differences were small. Consequently, it appears that pilots process information in very much the same manner for rotation in both directions about the three major body axes.

Although the power law with an exponent of 1.4 gives a good description of the data for these pilots, a detailed analysis of the results suggests that the perception of rotation may be more complex than implied by a single exponent. Systematic departures from a power law expressed by a single exponent are well known for a variety of experimental variables (Poulton, 1968; Stevens, 1970) and even within a given range of stimulus intensities (e.g., Miwa, 1968; Stevens, 1969). Similarly, an analysis of the pilots' data indicates that two straight lines with a much lower exponent for the five higher at values produce a more adequate description of the data than a single line. Independent least-squares analyses of the four lowest and the five highest at values for the three axes revealed consistent differences
(Table 2). Intersections of the two lines for the $x, y$, and $z$ axes were at the following at levels: 47,52 , and $56 \mathrm{deg} / \mathrm{sec}$, respectively. An analysis of Elsner's (1971) data indicates a similar, though less striking, break or knee in the data, and Miwa (1968) has described such an effect for vibration.

Stevens (1969), in discussing some of his data for tasting sucrose and sodium chloride that show similar deviations from a single power law, suggests that they may well be systematic departures from a general law. He says, "It is not known whether the flattening at the upper end represents a real effect, such as a saturating of the taste receptors, or whether it represents a reluctance of this particular group of $\mathrm{Os}$ to use numbers near and above 100." In the case of the pilots, it might be considered to be a threshold effect, but this notion can be discarded for two reasons. First, even the lowest acceleration was well above the thresholds of all of the pilots. 1 Second, the data (Fig. 1) fall on two lines rather than on a curve, which is typical of the threshold effect (Poulton, 1968). Consequently, it is suggested that for the perception of rotation, the effect may well be real.

Several lines of evidence regarding semicircular canal functioning suggest possible contributing factors that may lead to a reduction in the power law at the upper levels of acceleration. It has been known for some years that there are two major types of sensory cells in the crista ampullaris (Wersall \& Flock, 1965). Differential functioning of these two types of cells at different acceleration levels may contribute to such an effect. It is also possible that there may be a decreasing compression effect of cupular displacement on the hair cells across the surface of the crista at higher acceleration levels. This may reduce the increments in the total neurological effects and consequently reduce the magnitude estimates. This notion is supported by Dohlman's (1971) description of two types of cupular displacement. He describes a 
shearing motion at low levels of angular acceleration that leads to great sensitivity. The second type is a bending of the cupula as a whole at higher levels of acceleration. Finally, these magnitude estimates are a result of information from two sets of semicircular canals. For any given direction of rotation, the rate of firing on one side will increase while the other decreases (Wersäll \& Flock, 1965). At the higher levels of acceleration, the firing of one side will approach its minimum rate of firing and therefore contribute less to the summation effect. Further support for the notion of a differential functioning of the canals above $40-50$ at comes from the aftereffects of rotation. These aftereffects, as revealed in cupulograms, have been reported to deviate from a straight line function above at levels of about 50-60 (Groen \& Jongkees, 1948). Higher levels are avoided in clinical testing because they are considered to be nonphysiological and produce prolonged aftereffects. The particular mode of semicircular canal functioning that may produce such changes in exponents needs to be clarified by further investigation of the mechanical, physiological, and neurological systems involved.

In any event, for the wide range of at levels used in this experiment, it is suggested that the perception of rotation is more adequately described by two, rather than one, power laws.
The notion that such results are not merely an artifact of the magnitude estimation procedure is indirectly supported by Miwa's (1968) reports of different equations at different intensities of vibration. Exponents comparable to those exhibited by the pilots could be derived from Miwa's equations. His results are all the more significant since he used both bisection and fractionation rather than magnitude estimation and tested both hand and whole body vibration. However, definitive statements on this issue must await replication of these findings using other experimental conditions.

\section{REFERENCES}

CLARK, B., \& STEWART, J. D. Thresholds for the perception of angular acceleration about the three major body axes. Acta Otolaryngologica, 1970, 69, 231-238.

DOHLMAN, G. F. The attachment of the cupulae, otolith and tectorial membranes to the sensory cell areas. Acta Otolaryngologica, 1971, 71, 89-105.

ELSNER. W. The power laws for the perception of rotation and the oculogyral illusion. Perception \& Psychophysics, $1971,9,418-420$.

GROEN, J. J., \& JONGKEES, L. B. W. The turning test with small regulable stimuli. III. The advantages of cupulometry over the classic technique of Barany. Journal of Laryngology otology, 1948, 62, 231-235.

GUEDRY, F. E., JR., MONTAGUE, K. E. Quantitative evaluation of the vestibular Coriolis reaction. Aerospace Medicine, $1961,32,487-500$.
LANE, H. L., CATANIA, A. C., \& STEVENS, S. S. Voice level: Autophonic scale, perceived loudness, and effects of sidetone. Journal of the Acoustical Society of America, 1961, 33, 160-167. MIWA, T. Evaluation methods for vibration effect. Part 4. Measurements of vibration greatness for whole body and hand in vertical and horizontal vibrations. Industrial Health, 1968, 6, 1-10.

PARSONS, R. D. Magnitude estimates of the oculogyral illusion during and following angular acceleration. Journal of Experimental Psychology, 1970, 84, 230-238.

POULTON, E. C. The new psychophysics: Six models for magnitude estimation. Psy chological Bulletin, 1968, 69, 1-18.

REASON, J. T. \& GRAYBIEL A Magnitude estimations of Coriolis sensations. Report No. NAMI-1082. Pensacola, Fla: Naval Aerospace Medical Institute, 1969.

STEVENS, S. S. The direct estimation of sensory magnitudes-loudness. American Journal of Psychology, 1956, 69, 1-23.

STEVENS, $\boldsymbol{S}$. S. Sensory scales of taste intensity. Perception \& Psychophysics, $1969,6,302-308$.

STEVENS, $\mathbf{S}$. $\mathbf{S}$. Neural events and the psy chophysical law. Science, 1970, 170, 1043-1050.

STEWART, J. D., \& CLARK, B. Coriolis effects during pitch and roll maneuvers in a piloted flight simulator. Aerospace Medicine, 1965, 36, 105-112.

WERSÄLL, J., \& FLOCK, A. Functional anatomy of the vestibular and lateral line organs. In W. D. Neff (Ed.). Contributions to sensory physiology. New York: Academic Press, 1965.

\section{NOTE}

1. Clark, B., \& Stewart, J. D. Comparison of the sensitivity to rotation of pilots and nonpilots, in preparation.

(Accepted for publication February 7, 1972.) 